\title{
Fire Simulation and Evacuation Risk Analysis on Subway Train
}

\author{
Xiaoya Song ${ }^{1,2}$, Changxing $\mathrm{Ren}^{3}$, Fan $\mathrm{Wu}^{2}$, Qi Ni ${ }^{1}$, Jun Jiang ${ }^{2,4}$ \\ ${ }^{1}$ Firefighting Inspection Department, Taizhou Branch, Jiangsu Province Special Equipment Safety Supervision \\ Inspection Institute, Taizhou 225300, China \\ ${ }^{2}$ Nanjing Tech University, Jiangsu Key Laboratory of Hazardous Chemicals Safety and Control, Nanjing, \\ 210009, China \\ ${ }^{3}$ Tianjin Fire Research Institute of the Ministry of Public Security, Tianjin, 300381, China \\ ${ }^{4}$ Nanjing Boiler Pressure Vessel Inspection Institute, Nanjing, 210019, China

\section{地铁站列车火灾模拟及人员疏散风险分析} \\ 宋晓亚 ${ }^{1,2}$, 任常兴 ${ }^{3}$, 吴凡 ${ }^{2}$, 倪奇 ${ }^{1}$, 姜君 ${ }^{2,4}$ \\ ${ }^{1}$ 江苏省特种设备安全监督检验研究院泰州分院消防检测中心, 江苏泰州 225300 , 中国 \\ 2 南京工业大学江苏省危险化学品本质安全与控制技术重点实验室, 江苏南京 210009 , 中国 \\ 3 公安部天津消防研究所, 天津 300381 , 中国 \\ 4 南京市锅炉压力容器检验研究院, 江苏南京 210019 , 中国
}

\begin{abstract}
FDS+EVAC was used to simulate the subway train fire and research the related risk analysis of human evacuation. It was also used to demonstrate the feasibility and effectiveness of emergency evacuation on subway fire. The baggage in the middle of train burned out in the simulation and the power of fire get the peak of $7.5 \mathrm{MW}$. And the content of carbon monoxide and carbon dioxide and the temperature distribution were analyzed. Moreover, the people number-time change in each compartment and platform in the given fire scenarios was described and simulated, and the critical factors and criterion on people evacuation were put forward. The results can provide some references for the study of smoke spread and human evacuation.
\end{abstract}

Keywords: FDS+EVAC; Subway; Train Fire; Personnel Evacuation; Risk Analysis

\section{摘要}

本文通过火灾动力学软件 FDS+Evac 对某地铁站列 车火灾情况下人员疏散问题进行模拟研究及风险分 析, 论证该地铁站火灾应急疏散预案的可行性及有效
性。模拟方案为列车中部行李发生火灾, 火源功率为 $7.5 \mathrm{MW}$ 。通过分析相同时间点的车厢中部 $\mathrm{CO} 、 \mathrm{CO}_{2}$ 含量以及温度的分布情况, 观察火灾发生后每节车厢 内及站台层人数随时间的变化, 明确影响火灾情况下 人员疏散的限制条件。模拟结果为烟气蔓延规律及人 员疏散研究提供相关参考。

关键词: FDS+EVAC; 地铁站; 列车火灾; 人员疏散; 风险分析

1.引言

随着城市轨道交通的飞速发展, 地铁凭其快捷、方便、 准时、舒适、无污染等诸多优点, 已成为大型城市重 要交通工具之一 ${ }^{[1,2]}$ 。地铁火灾问题一直是消防工作 者和学者关注的热点和难点, 火灾风险管控存在诸多 难题 ${ }^{[3]}$ 。

地铁一旦发生火灾, 容易形成热气浪和浓烟, 同 时产生大量有毒有害气体, 而烟气扩散与人员疏散的 方向一致, 再加上空间密闭、通道狭长等特性, 被困 人员容易出现恐慌心理, 安全疏散难度大, 因此有必 要对地铁站列车火灾情况下有毒有害烟气的蔓延规 律及人员疏散风险进行深入研究 ${ }^{[4-7]}$ 。

李朋等 ${ }^{[1]}$ 针对地铁隧道区间火灾的火势蔓延迅 速等特点, 通过优化疏散路径等方法优化了地铁隧道 
Risk Analysis and Crisis Response in Big Data Era (RAC-16)

区间安全疏散措施。李意等 ${ }^{[2]}$ 采用 FDS 软件模拟地铁 (岛式) 车站火灾, 分析发现, 全部人员疏散在 $165 \mathrm{~s}$ 完成。陈同刚等 ${ }^{[3]}$ 采用 FDS 软件模拟地铁控制中心火 灾, 利用 Pathfinder 软件模拟紧急情况下人员疏散, 结果表明, 监控大厅设置成独立的防火分区及采用防 火墙分隔可有效阻止火势蔓延, 采用借用疏散口设计 能够保证人员安全疏散到室外安全区域。王春雷等 ${ }^{[4]}$ 开展了应急疏散试验并基于此建立优化模型, 结果表 明, 地铁应急疏散恐慌程度受年龄、接受安全教育程 度等个体因素, 以及人员密度、疏散环境复杂程度、 事故发生位置等环境因素影响。展望等 ${ }^{[5]}$ 采用 FDS 软件模拟地铁车站的烟气流场和细水雾的相互作用, 结果表明, 当细水雾施加角度为 $60^{\circ}$ 时抑烟效果最 好。汪志雷等 ${ }^{[6]}$ 采用 FDS+EVAC 软件对地铁列车区 间隧道火灾进行模拟, 结果表明, 地铁在 $2700 \mathrm{~m}$ 长 的隧道中发生火灾时进站疏散方式所需的时间更短。
本文立足于轨道交通的功能和结构特点, 采用 火灾动力学软件 FDS+Evac 对某地铁站列车火灾情 况下人员疏散问题进行模拟研究及风险分析。

\section{2.数值模拟}

\section{1. 火灾数值模拟}

\subsection{1.模型与边界条件}

本次模拟简化了地铁站模型, 对站台与站厅层的一些 附属设备 (如指示牌, 垃圾桶等) 不予考虑。火灾时 仅考虑开启地铁站内相应的通风与排烟设备, 不考虑 消防水系统的影响。地铁站及列车模型示意图见图 1 , 具体的模型尺寸见表 1 。

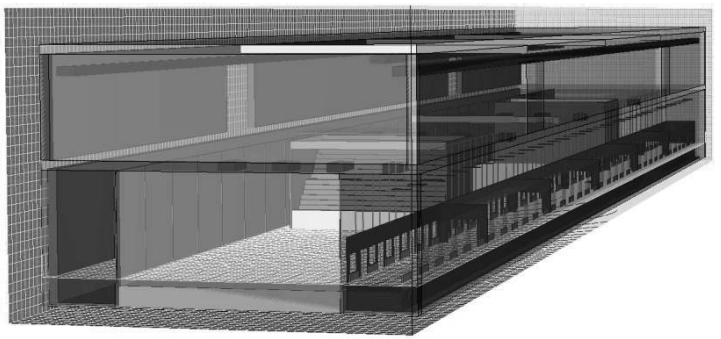

图 1 地铁站及列车模型示意图

表 1 地铁构筑物尺寸

\begin{tabular}{|c|c|c|c|c|}
\hline 双层岛式车站 & 长(m) & 宽(m) & 高(m) & 备注 \\
\hline 站厅层 & 120 & 20 & 5.5 & 位于地上 1 层 \\
\hline 站台层 & 120 & 20 & 6 & 位于地下 1 层 \\
\hline 地铁出入口 & -- & 5.5 & 4.5 & 位于站厅层, 左右两侧各有 2 个 \\
\hline 楼梯 & 6 & 10 & 5.5 & $\begin{array}{l}\text { 连接站台层与站厅层, } 2 \text { 个, 间距 } \\
30 \mathrm{~m}\end{array}$ \\
\hline 地铁隧道口 & -- & 5 & 6 & 位于站台层 \\
\hline 挡烟垂壁 1 & -- & -- & 0.5 & 用于防烟分区, 4 个 \\
\hline 挡烟垂壁 2 & \multicolumn{3}{|c|}{ 其下缘至楼梯踏步面的垂直距离为 $4.5 \mathrm{~m}$} & 楼梯口处, 每个楼梯 3 个 \\
\hline 排风口 & 1 & 0.5 & -- & -- \\
\hline
\end{tabular}

模拟中设置在列车中部的火源使用了简单热解 模型 ${ }^{[8]}$, 热解材料为 $\mathrm{PVC}$, 功率为 $7.5 \mathrm{MW}$, 功率曲 线为 $\mathrm{t}^{2}$ 超快速火, 功率增长系数 $\alpha$ 为 0.19 。由于列车 火灾产生的烟气主要是通过轨顶排烟系统排出, 因此 根据规范计算出排烟量, 轨顶排烟口的排烟风速为 $3 \mathrm{~m} / \mathrm{s}$, 每节车厢的上方都有 6 个轨顶排烟口。

\subsection{2. 模拟结果分析}

人员逃生的环境判断标准主要包括: 人员特征高度处 的烟气温度不超过 $60^{\circ} \mathrm{C}, \mathrm{CO}$ 浓度不超过 $500 \mathrm{ppm}$, $\mathrm{CO}_{2}$ 浓度不超过 $2 \%$ 。

不同时间点车内温度分布情况见图 2。不同时间 点车内 $\mathrm{CO}$ 分布情况见图 3。不同时间点车内 $\mathrm{CO}_{2}$ 分 


\section{Risk Analysis and Crisis Response in Big Data Era (RAC-16)}

布情况见图 4。360s 时楼梯口不同高度处的风速分布 见图 5。

由图 2 可以看出, 列车火灾发生后, 温度最高的 区域集中在火源上方的几个轨顶排烟口, 说明轨顶排 烟起到了很好的效果，不但有效抑制了烟气的蔓延， 而且控制了烟气的最高温度。除了着火车厢以及相邻 车厢, 其余区域的温度都接近于环境温度, 这对人员 的安全疏散非常有利。

由图3可以看出, $\mathrm{CO}$ 浓度最高的区域为 $7 \mathrm{ppm}$,

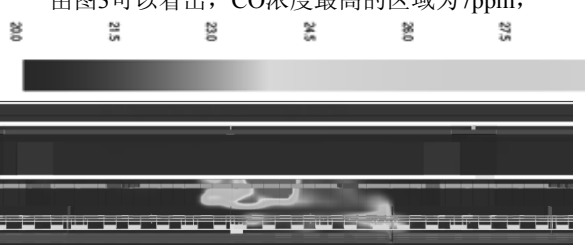

(a) $120 \mathrm{~s}$ 时列车中部温度分布云图

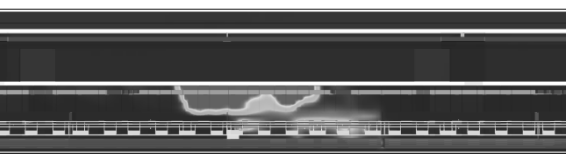

(c)360s 时列车中部温度分布云图

图 2 不同时间点车内温度分布情况

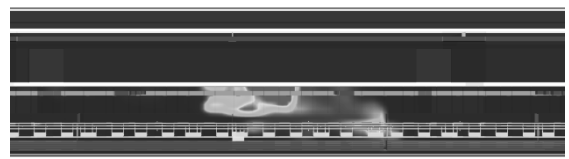

(a) $120 \mathrm{~s}$ 时列车中部 CO 分布云图

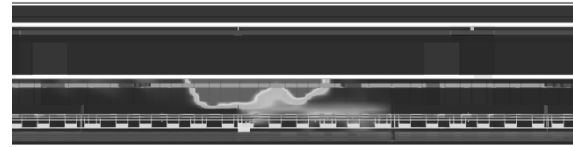

(c) $360 \mathrm{~s}$ 时列车中部 $\mathrm{CO}$ 分布云图

图 3 不同时间点车内 $\mathrm{CO}$ 分布情况

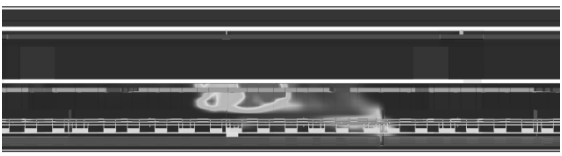

(a) $120 \mathrm{~s}$ 时列车中部 $\mathrm{CO}_{2}$ 分布云图

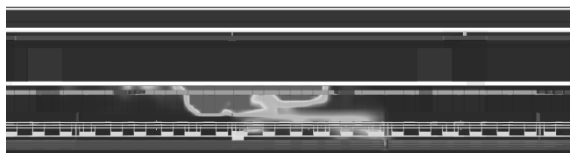

(b) $240 \mathrm{~s}$ 时列车中部 $\mathrm{CO}$ 分布云图

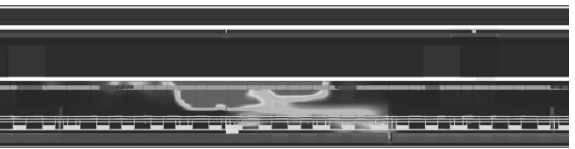

(b) $240 \mathrm{~s}$ 时列车中部 $\mathrm{CO}_{2}$ 分布云图

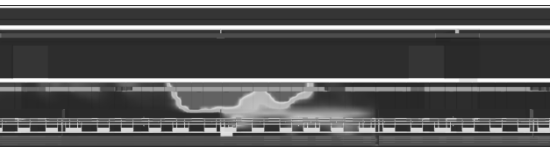

(c) $360 \mathrm{~s}$ 时列车中部 $\mathrm{CO}_{2}$ 分布云图

图 4 不同时间点车内 $\mathrm{CO}_{2}$ 分布情况 
Risk Analysis and Crisis Response in Big Data Era (RAC-16)

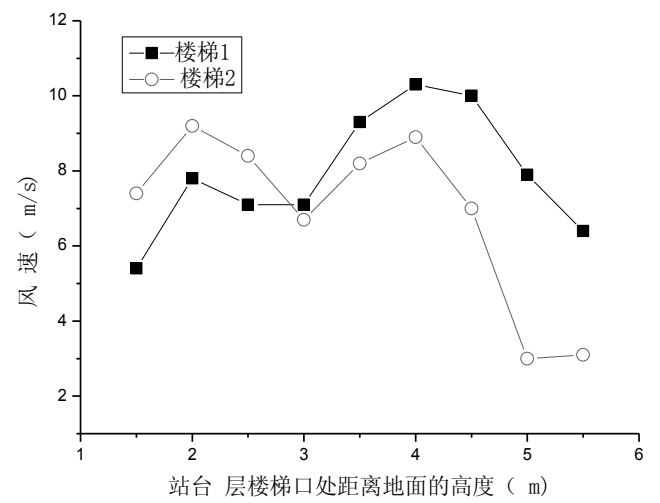

图 $5360 \mathrm{~s}$ 时楼梯口不同高度处的风速分布

\section{2.人员疏散数值模拟}

人员疏散安全性能化评估主要取决于两个特征时间: 可用安全疏散时间（ASET）和必需安全疏散时间 ( RSET ) ${ }^{[10]}$ 。根据《地铁设计规范》 （GB50157-2013），第28.2.11 条规定，在远期或客 流控制期超高峰小时发生火灾时, 要在 $6 \mathrm{~min}$ 内将一 列列车的乘客和站台上候车人员撤离至安全区, 即 ASET 为 $6 \mathrm{~min}^{[12]}$ 。第 28.2.12 条规定, 对高度不超过 3 层的车站, 乘客从站台疏散至站厅或其他安全区域 时间（即 RSET）计算见公式 (1) ${ }^{[11,12] 。}$

$$
\text { RSET }=1+\frac{\mathrm{Q}_{1}+\mathrm{Q}_{2}}{0.9\left[A_{1}(N-1)+A_{2} B\right]} \leq \operatorname{ASET}(6 \text { min }) \quad(1)
$$

式中, $\mathrm{Q}_{1}$ 为列车载客人数; $\mathrm{Q}_{2}$ 为站台候车人

数; $A_{1}$ 为一台自动扶梯通过能力; $A_{2}$ 为步行楼梯通

过能力; $N$ 为自动扶梯台数; $B$ 为步行楼梯总宽度。 模拟中设置火灾前站台层人员总数为 600 人, 其 中老人 120 人, 儿童 60 人, 男女青年分别 210 人; 设置 列车内人员总数为 759 人, 其中老人 150 人, 儿童 75

人, 男女青年分别 268 人与 266 人。

不同时间点站台层与列车人员分布情况见图6。 火灾时站台层与列车总人数及进入站台层楼梯口人 数随时间变化见图7。疏散过程中死亡人数随时间变 化见图8。

由图6可以看出, 在不到 $2 \mathrm{~min}$ 的时间内, 全部人 员疏散完毕。虽然靠近火源区的部分人员在疏散过程
中受到了烟气影响, 但由之前的火灾模拟分析可知, 烟气未对疏散人员造成伤害。

由图7、图8可以看出, $150 \mathrm{~s}$ 时站台层与列车上的 所有人员疏散完毕并且从两个楼梯口疏散的人数大 致相同。虽然从烟气分布上来看, 从楼梯口2 (右侧) 处疏散的风险似乎更大一些, 但由于右侧烟气的影响 在人员可容忍范围内, 因此大多数疏散人员不会改变 原有的疏散方向, 这也是站台层两侧楼梯口疏散人员 数大致相同的原因。同时, 由图8可以看出, 整个疏 散过程没有人员死亡。这个结论与之前的火灾模拟分 析是一致的, 无论是烟气温度还是 $\mathrm{CO}$ 浓度、 $\mathrm{CO}_{2}$ 浓度, 均未对人员疏散造成威胁。

\section{3.总结}

通过对模拟结果的分析发现: 该地铁站列车火灾发生 后, 轨顶排烟系统、楼梯口送风系统起到了很好的效 果, 不但有效抑制了烟气的蔓延, 而且控制了烟气的 最高温度。同时, $\mathrm{CO}$ 浓度、 $\mathrm{CO}_{2}$ 浓度未对人员疏散 造成威胁。整个疏散过程没有人员死亡。由此可见: (1) 该地铁站列车火灾情况下的人员疏散方案是安全 可行的。(2) 该车站的通风排烟等消防设施设置及布 局合理, 能够保证火灾情况下有毒有害气体浓度和烟 气温度、烟气层高度满足人员疏散要求。

通过与李意等 ${ }^{[2]}$ 建立的地铁车站火灾模型比较, 可以 看出, 本文优势在于更加系统性研究了火灾时人员分 布情况 (不同时间点站台层与列车)、人数随时间变 化情况（站台层与列车内及进入站台层楼梯口）、 
Risk Analysis and Crisis Response in Big Data Era (RAC-16)

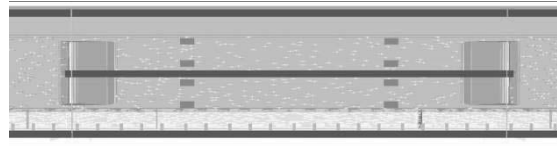

(a) 火灾发生前站台层与列车人员分布图

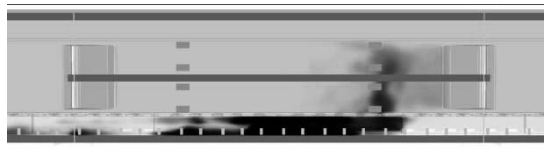

(c)火灾发生后 $240 \mathrm{~s}$ 时站台层与列车人员分布图

图 6 不同时间点站台层与列车人员分布情况

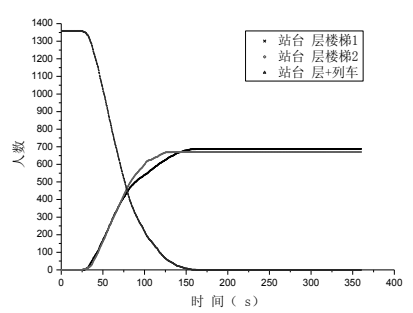

图 7 火灾时站台层与列车总人数及进入站台层楼梯口人数随时间变化图 死亡人数随时间变化情况（疏散过程中); 同时, 本 文在样本数（1359 人, 站台层、列车内）明显多于 李意等 ${ }^{[2]}$ 模型的样本数 (450人, 站台层、站厅层) 情况下人员疏散时间明显较少, 又列车内人员疏散较 站台层、站厅层的难度更大, 由此可见, 本文所建的 地铁站火灾模型在稳定性、应用范围等方面具有一定 的优越性。

本次模拟研究为地铁站应急疏散预案的制定、防 火设计的优化及消防安全风险的控制提供了建议和 理论依据。

\section{参考文献}

[1] 李朋. 地铁隧道区间火灾特点及安全疏散方式探 讨. 现代城市轨道交通, (1):58-62, 2016.

[2] 李意. 地铁车站站台火灾性能指标的数值模拟与 分析. 交通与运输, (12):126-129, 2015.

[3] 陈同刚, 尼华, 肖鹏让等. 某地铁控制中心防火 设计难点分析. 消防科学与技术, 35(1):73-75, 2016.

[4] 王春雪, 索晓, 吕淑然等. 地铁应急疏散恐慌程 度模型研究. 中国安全科学学报, 25(2):171-176, 2015.

[5] 展望, 蒋军成, 孙志影等. 细水雾对地铁火灾烟 气抑制的数值模拟. 建筑科学, 30(7):103-106, 2014. 2014. 178-187, 2012. 2002. 划出版社.

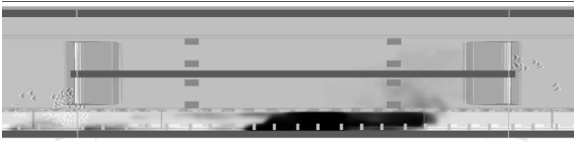

(b) 火灾发生后 $120 \mathrm{~s}$ 时站台层与列车人员分布图

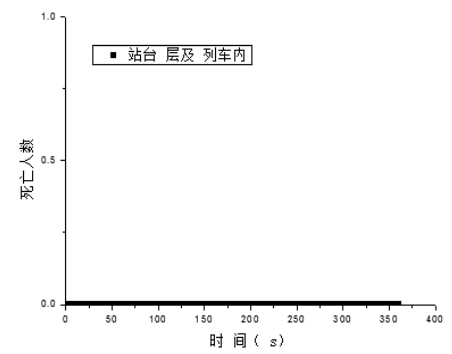

图 8 疏散过程中死亡人数随时间变化图

[6] 汪志雷, 华敏, 徐大用等. 地铁隧道火灾人员疏 散模拟研究. 消防科学与技术, 33(6): 645-648,

[7] J. Ma, S. B. Liu, W. L. Wang, et al. GIS-based micro-simulation queue model for vehicle evacuation. Risk Analysis and Crisis Response, 2(3):

[8] Standard of Smoke and Heat Venting. NFPA 204M,

[9] Quincy, Mass. NFPA 92B, Guide for smoke management system in malls, atria and large area. National Fire Protection Association, 1995.

[10] P. Yang, C. Li, D. Chen. Fire emergency evacuation simulation based on integrated fire evacuation model with discrete design method. Advances in Engineering Software, 65:101-111, 2013.

[11] GB50157-2013, 地铁设计规范. 北京: 中国计

[12] 赵刚, 李炎锋, 崔彦强. 中庭式地铁站疏散时间 计算与比较分析. 消防科学与技术, 35(3):329-331,2016. 